Check for updates

Cite this: RSC Adv., 2019, 9, 6965

Received 9th December 2018

Accepted 18th February 2019

DOI: $10.1039 / c 8 r a 10113 b$

rsc.li/rsc-advances

\title{
Hollow ZnO microspheres functionalized with electrochemical graphene oxide for the photodegradation of salicylic acid $\dagger$
}

\author{
Marcos Rodríguez Hernández, ${ }^{a}$ Arxel de León Santillán, (D)*bc Edgar de Casas Ortiz, ${ }^{a}$ \\ Salvador Fernández Tavizón, ${ }^{a}$ Ivana Moggio, (D) a Eduardo Arias, ${ }^{a}$ \\ Carlos Alberto Gallardo-Vega, ${ }^{a}$ Jesús Alfonso Mercado Silva ${ }^{a}$ \\ and Enrique Díaz Barriga-Castro (D) ${ }^{a}$
}

\begin{abstract}
Hollow ZnO microspheres were successfully synthesized by a hydrothermal method and then functionalized with graphene oxide (GO) flakes, previously obtained through electrochemical oxidation. Their photocatalytic activity toward the photodegradation of salicylic acid under UV light irradiation was evaluated by UV-Vis spectroscopy. Unfunctionalized microspheres and ZnO functionalized with chemically oxidized graphene were also studied as comparative terms. The hybrid materials of $\mathrm{ZnO}$ with both electrochemical and chemical GO gave a similar photodegradation yield of $\sim 28 \%$ against $18 \%$ of the non-functionalized microspheres. The similar degradation yields and rate constants obtained with the two GO synthetic methods indicate that electrochemical oxidation of GO represents an eco-friendly option over traditional methods for photocatalytic degradation systems.
\end{abstract}

\section{Introduction}

One of the principal pollutants that comes from pharmaceutical, food and cosmetic industries is salicylic acid (SA). Despite the fact that several decomposition methods have been proposed, the photodegradation of this contaminant is an easy and ecological method for industrial wastewater treatment. ${ }^{1}$

Photodegradation of compounds requires, in general, the excitation of a semiconductor, such as tin or zinc oxide. $\mathrm{ZnO}$ has a low production cost, high photosensitivity, and nontoxicity. Its photocatalytic activity has been studied for different morphologies: nanorods, nanodiscs, and hollow microspheres. ${ }^{2,3}$ These latter have low density and high surface/volume ratio that allow higher efficiency in charge recollection and charge transport with respect to bulk ZnO., Nevertheless, as the band gap of this metal oxide is quite large (3.37 eV), further improvement in the photocatalytic activity has been reported by making composites with carbon-based materials. ${ }^{6}$ In particular, graphene oxide (GO) presents

${ }^{a}$ Centro de Investigación en Química Aplicada, Boulevard Enrique Reyna 140, 25294 Saltillo, Coahuila, Mexico

${ }^{b}$ CONACYT-Centro de Investigación en Química Aplicada, Boulevard Enrique Reyna 140, 25294 Saltillo, Coahuila, Mexico.E-mail: arxel.deleon@ciqa.edu.mx

${ }^{c}$ Research Group of Optical Properties of Materials (GPOM), Centro de Investigaciones en Óptica, A.P. 1-948, 37150 León, Gto., Mexico

$\dagger$ Electronic supplementary information (ESI) available. See DOI: 10.1039/c8ra10113b different functional groups such as carboxylic acid, hydroxyl, and epoxide groups on its two-dimensional surface that allows functionalization with metal oxides. $^{7-10}$ Additionally to improve the band gap increasing the exciton generation, GO effectively transports the charges to the surface of $\mathrm{ZnO}$ nanostructures. $^{10}$ GO is usually obtained through chemical methods such as Brodie, Staudenmaier, Hummers, and Marcano, ${ }^{11-14}$ which require the use of toxic and hazards materials and involve difficult procedures for their purification. Conversely, the electrochemical method is more straightforward, eco-friendly and economical. ${ }^{15-17}$

In this paper, we report on the synthesis of hollow microspheres of zinc oxide and his surface modification with graphene oxide flakes obtained electrochemically, as well as their photocatalytic activity towards the degradation of salicylic acid. ZnO microspheres without GO and with GO obtained by a chemical method (through a modification of Marcano procedure) were also studied as comparative terms. It is to be pointed out that, during the preparation of this manuscript, a work on GO-ZnO microspheres was published. Nevertheless, the syntheses of both, the graphene oxide and the functionalized $\mathrm{ZnO}$ hybrid are different and in that work, the photocatalytic activity was investigated in the degradation of methylene blue. ${ }^{18}$

The results here reported demonstrate that electrochemical oxidation of graphene can be an eco-friendly option over traditional methods to obtain $\mathrm{ZnO}$ microsphere hybrids with enhanced photocatalytic activity. 


\section{Experimental details}

Materials

Ethylene glycol, hydrogen peroxide at $30 \%$, sulfuric, phosphoric, and hydrochloric acid were purchased by Fermont, zinc acetate dihydrate, salicylic acid, and graphite bar were acquired from Aldrich Chemical Company, graphite powder 3160 was supplied by Asbury Carbons graphite. Anhydrous dextrose and potassium permanganate were obtained from Analytyka.

\section{Electrochemical synthesis of graphene oxide}

The electrochemical oxidation was carried out in an air power supply at $15 \mathrm{~V}$ and $1.5 \mathrm{amp}(22.5 \mathrm{~W})$ in a VersaSTAT3 Potentiostat-Galvanostat from Ametek Scientific Instruments. The working electrode was a graphite bar and platinum wire as the second electrode. Measurements were performed at room temperature, using ammonium carbonate solution $0.25 \mathrm{M}$ as the supporting electrolyte was at reaction times of 7 hours. The product was washed three times with both, deionized water and industrial grade ethanol. In the following, the product of this reaction will be referred to as $\mathrm{GO}-\left(\mathrm{NH}_{4}\right)_{2} \mathrm{CO}_{3}$.

\section{Chemical synthesis of graphene oxide}

Chemical oxidation of graphite was carried out according to Marcano et al. ${ }^{11}$ Briefly, $640 \mathrm{~mL}$ of sulfuric acid, $90 \mathrm{~mL}$ of phosphoric acid and $6 \mathrm{~g}$ of Asbury 3160 graphite were put in a one neck flask provided with mechanical stirring. The reactive mixture was cooled to $0{ }^{\circ} \mathrm{C}$. Then, $36 \mathrm{~g}$ of $\mathrm{KMnO}_{4}$ were added, and the temperature increased to $60^{\circ} \mathrm{C}$. The reaction was left for 4 hours. Finally, a solution of $20 \mathrm{~mL}$ of hydrogen peroxide $(30 \%)$ in $200 \mathrm{~mL}$ of deionized water was added. The mixture was centrifuged and washed with $200 \mathrm{~mL}$ of $\mathrm{HCl}$, $200 \mathrm{~mL}$ of ethanol and subsequently with deionized water until $\mathrm{pH}$ 7. In the following, the product of this reaction will be referred as GO.

\section{Synthesis of hollow ZnO microspheres}

Hollow $\mathrm{ZnO}$ microspheres were synthesized by a modified methodology of Seyed et al. ${ }^{19}$ Carbon microspheres (CM) were first synthesized as following: $15 \mathrm{~g}$ of anhydrous dextrose dissolved in a mixture of $15 \mathrm{~mL}$ of ethylene glycol and $35 \mathrm{~mL}$ of deionized water were put into a Teflon-lined stainless steel autoclave and left to react during 4 hours at $180{ }^{\circ} \mathrm{C}$. The product was then washed three times with deionized water and dried at $80^{\circ} \mathrm{C}$ for 18 hours. After this time, $1 \mathrm{~g}$ of carbon microspheres, $2.2 \mathrm{~g}$ of zinc dehydrate acetate and $60 \mathrm{~mL}$ of deionized water were added to the autoclave. The solution was heated for $90 \mathrm{~min}$ at $120{ }^{\circ} \mathrm{C}$. The product was then rinsed with deionized water, ethanol and finally pyrolyzed at a heating ramp of $1{ }^{\circ} \mathrm{C} \mathrm{min}^{-1}$ from 0 to $500{ }^{\circ} \mathrm{C}$; maintained at this temperature for 4 hours. In the following, the product of this reaction will be referred to as ZnO.

\section{Preparation of graphene oxide supported on ZnO hollow microspheres}

In a vessel, $30 \mathrm{mg}$ of the previously synthesized graphene oxide was dissolved in a mixture of $70 \mathrm{~mL}$ of isopropanol and $50 \mathrm{~mL}$ of deionized water and sonicated for 2 hours. In another vessel, $90 \mathrm{mg}$ of $\mathrm{ZnO}$ hollow microspheres were suspended in $40 \mathrm{~mL}$ of deionized water, and then this solution was transferred to the vessel containing the GO. The mixture was kept under magnetic stirring for one hour. Finally, the material was filtered off with PTFE membranes. The product of electrochemical (chemical) $\mathrm{GO}$ with $\mathrm{ZnO}$ will be referred to as $\mathrm{GO}-\left(\mathrm{NH}_{4}\right)_{2} \mathrm{CO}_{3} / \mathrm{ZnO}(\mathrm{GO} /$ $\mathrm{ZnO})$.

\section{Characterization}

Raman spectra were recorded on a micro Raman Xplora by Horiba Scientific, by exciting at $532 \mathrm{~nm}$ with a nanoLED of 25 $\mathrm{mW}$ (attenuation filter of 10\%). ATR Fourier transformed infrared (FTIR) analysis was carried out in Nicolet iS5 Thermo scientific iD7ATR, in the range from $4000-600 \mathrm{~cm}^{-1}, 16$ resolutions, 64 scans, using germanium as reference standard material. SEM characterization was realized with a JEOL JSM7041F. The samples were gold-palladium sputter coated. The GO morphology was determined by transmission electron microscopy (TEM) with FEI Titan microscope at $300 \mathrm{kV}$, by casting few drops of a dispersion of GO in water $\left(0.1 \mathrm{mg} \mathrm{mL}^{-1}\right)$ on a Lacey Carbon grid. Thermogravimetric analysis (TGA) was carried out using TA Instruments TGA Q500, at a heating rate $10{ }^{\circ} \mathrm{C} \mathrm{min}{ }^{-1}$ from 25 to $600{ }^{\circ} \mathrm{C}$ in nitrogen, and from $600{ }^{\circ} \mathrm{C}$ to $800{ }^{\circ} \mathrm{C}$ in oxygen. The X-ray diffraction (XRD) study of the powder materials was performed on an Eco D8 Advance of Bruker. The data were recorded in the range of $3-90^{\circ} 2 \theta$, at a rate $0.01^{\circ} \min ^{-1}, 40 \mathrm{kV}$ voltage and $25 \mathrm{~mA}$ of emission current. XPS analysis was realized on a PHI VersaProbe II instrument of Physical Electronics, during the acquisitions the analysis chamber was a $2 \times 10-8$ mTorr. A monochromatic Xray source with an aluminium anode and radiation energy of $1486.6 \mathrm{eV}$ was used. Survey spectra were obtained with an energy step of $117.4 \mathrm{eV}$, between 1400-0 (eV) of energy binding. High resolution scans of $\mathrm{C} 1 \mathrm{~s}, \mathrm{O} 1 \mathrm{~s}$ and $\mathrm{Zn} 2 \mathrm{p}_{3 / 2}$ were obtained with $11.75 \mathrm{eV}$ step. Gaussian deconvolution process was made with restrains only on FWHM; maximums and area being free.

\section{Photocatalytic activity}

Photocatalytic reactions were carried out in a home-made photoreactor under UV light of $20 \mathrm{~W}$ positioned horizontally above the liquid surface. Briefly, $10 \mathrm{ppm}$ of salicylic acid and 60 ppm of the previously synthesized material ( $\mathrm{ZnO}$; GO/ZnO; GO- $\left.\left(\mathrm{NH}_{4}\right)_{2} \mathrm{CO}_{3} / \mathrm{ZnO}\right)$, were collocated in a precipitate baker of $250 \mathrm{~mL}$ containing a magnetic stirrer and a peristaltic pump into the reactor. The photodegradation was monitored every 30 min during 4 hours by UV-Vis spectroscopy with a Cintra 2020 GBC spectrophotometer, following the change in the absorbance at the maximum wavelength peak of the salicylic acid (295 nm), using deionized water as the reference. 


\section{Results and discussion}

\section{Electrochemical synthesis of graphene oxide}

The electrochemical oxidation of graphite is evidenced after 15 min of reaction by the appearance of bubbles in the graphite surface electrode and by a colour change from transparent to the dark brown of the suspension. The graphite expands gradually, while the surface becomes rough and easily dilated. During the oxidation process, the presence of bubbles into the graphite layer's allows surpassing the van der Waals forces in the adjacent layers and the exfoliation of graphene layer occurs.

The oxidation of graphite, achieved by both electrochemical and chemical methods, is confirmed by vibrational spectroscopy, ATR-FTIR (Fig. 1a) and Raman (Fig. 1b). The infrared spectrum of the chemically oxidized graphene (Fig. 1a, solid line) is quite similar to that found in literature, ${ }^{\mathbf{2 0 , 2 1}}$ and presents the typical broad $\mathrm{OH}$ stretching band in the region of 3600$3000 \mathrm{~cm}^{-1}$ indicative of hydroxyls and carboxylic groups. The signal at $1730 \mathrm{~cm}^{-1}$ is assigned to the $\mathrm{C}=\mathrm{O}$ stretching vibration of the carboxylic group, while the band at $1632 \mathrm{~cm}^{-1}$ is due to $\mathrm{C}=\mathrm{C}$ mode of the carbonic skeleton of un-oxidized graphite. The peak at 1064 at $\mathrm{cm}^{-1}$ is consistent with the $\mathrm{C}-\mathrm{O}$ stretching. The band at $907 \mathrm{~cm}^{-1}$ are assigned to the different wagging
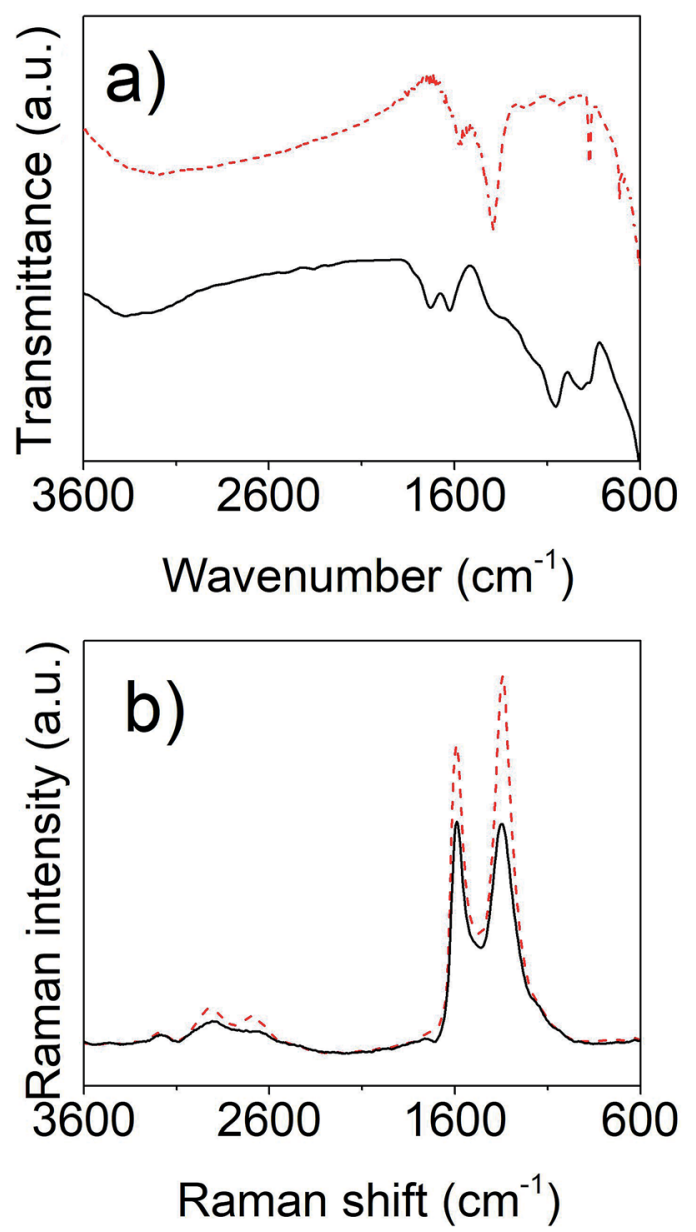

Fig. 1 FT-IR (a) and Raman (b) spectra of chemically (solid lines) and electrochemically (dashed lines) oxidized graphite. vibrations of the $\mathrm{OH}$, epoxy, ether, etc. vibrations. A bit different is the spectrum of the electrochemically obtained graphene oxide (Fig. 1a, dashed line). The $\mathrm{OH}$ stretching mode becomes broader ranging from 3600 up to $1600 \mathrm{~cm}^{-1}$, likely due to the $\mathrm{OH}$ and $\mathrm{NH}$ overlapped signals. The $\mathrm{C}=\mathrm{O}$ stretching vibration downshifts to $1577 \mathrm{~cm}^{-1}$ (and the corresponding C-O stretching to $1054 \mathrm{~cm}^{-1}$ ). A sharp peak appears at $1391 \mathrm{~cm}^{-1}$ that can be due to $\mathrm{OH}$ bending. The $\mathrm{C}=\mathrm{C}$ graphitic vibration cannot be observed. (ii) Raman spectra of both materials show the typical graphene oxide bands: the $\mathrm{G}$ band of the $\mathrm{E}_{2 \mathrm{~g}}$ vibration mode of the in-plane bond $\mathrm{C}=\mathrm{C}$ stretching motion of pairs of $\mathrm{C} \mathrm{sp}^{2}$ atoms $\left(1591 \mathrm{~cm}^{-1}\right)$ and the $\mathrm{D}$ breathing mode band of $\mathrm{A}_{1 \mathrm{~g}} \mathrm{C} \mathrm{sp}^{3}$ atoms $\left(\sim 1350 \mathrm{~cm}^{-1}\right)$, which relative intensity $I_{\mathrm{D}} / I_{\mathrm{G}}$ is 0.98 for the chemical oxidation and 1.23 for electrochemical oxidation, suggesting a larger number of defects in the latter material, consistent with the lack of detection of the $\mathrm{C}=\mathrm{C}$ vibration in the IR spectrum. ${ }^{22}$

The thermogravimetric analysis for the oxides (Fig. 2), shows a similar weight loss of around $10 \%\left(7.44 \%\right.$ for GO- $\left(\mathrm{NH}_{4}\right)_{2} \mathrm{CO}_{3}$ and $9.51 \%$ for GO) between $25{ }^{\circ} \mathrm{C}$ and $70{ }^{\circ} \mathrm{C}$, which is likely associated with desorption of the absorbed water. Between $70{ }^{\circ} \mathrm{C}$ and $184{ }^{\circ} \mathrm{C}$, a further weight loss of $11.79 \%$ for GO$\left(\mathrm{NH}_{4}\right)_{2} \mathrm{CO}_{3}$ and of $29.49 \%$ for $\mathrm{GO}$ is observed giving a total weight loss of $19.23 \%$ and $39.00 \%$ for GO- $\left(\mathrm{NH}_{4}\right)_{2} \mathrm{CO}_{3}$ and GO respectively.

This latter is often ascribed to pyrolysis of the labile oxygen containing functional groups, yielding $\mathrm{CO}, \mathrm{CO}_{2}$, and steam., ${ }^{\mathbf{1 , 6}, 23}$ The fact that a higher weight loss is observed for GO confirms that the chemical method yields to a larger extent of oxidation with respect to the electrochemical one. The final mass loss at $600{ }^{\circ} \mathrm{C}$ is attributed to the sublimation or burning of the damaged graphite regions or the decomposition of the carbon squeleton. ${ }^{24-26}$

Fig. 3 shows the X-ray diffractograms of chemically and electrochemically oxidized graphite. It can be seen, that the diffractogram of the electrochemical product presents a sharp reflection at $2 \theta=26.5^{\circ}$, which is at the same position of that of the graphite (as observed in the reference spectrum) and that

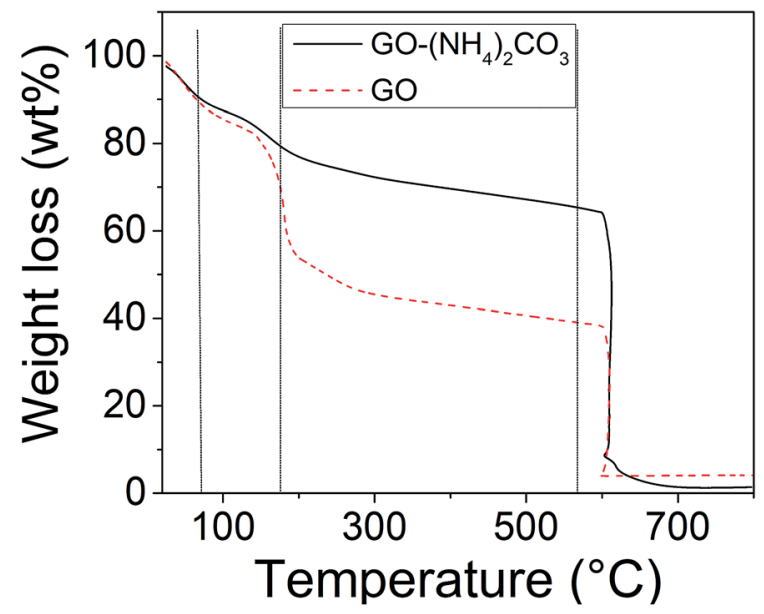

Fig. 2 TGA curves of chemically (solid lines) and electrochemically (dashed lines) oxidized graphite. Dotted lines are guides for the eyes. 


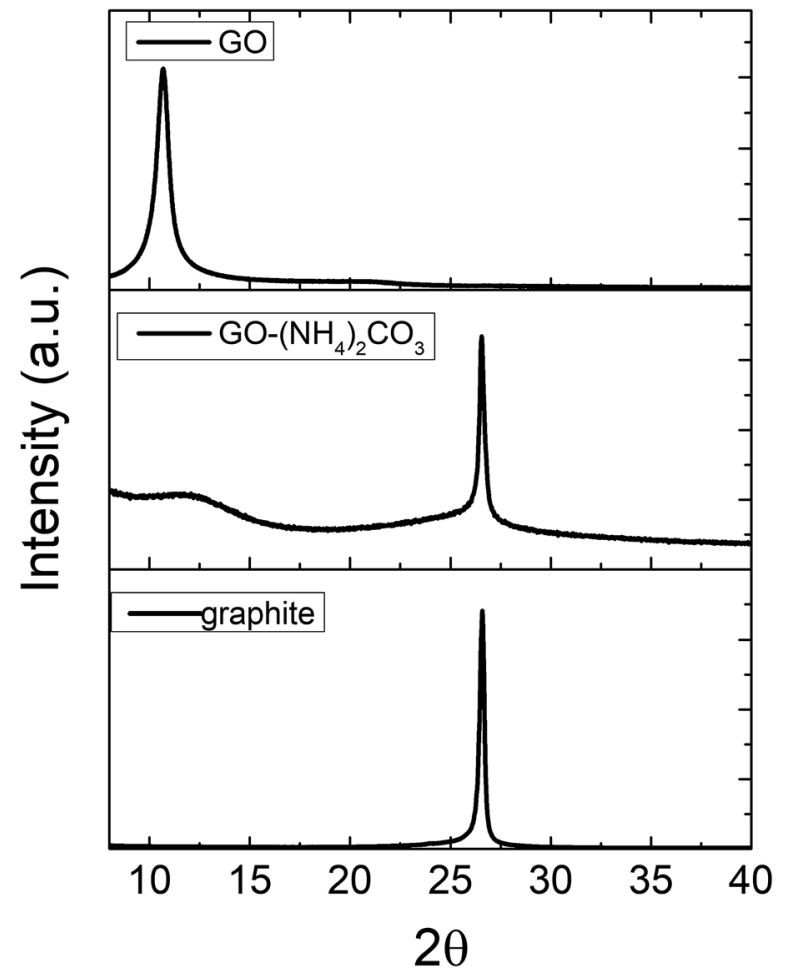

Fig. 3 X-ray diffractograms of chemically and electrochemically oxidized graphite. Graphite XRD spectrum is included for sake of comparison.

corresponds to (002) plane of graphite with an interplanar distance of $0.33 \mathrm{~nm}$. A second broadband is observed at $2 \theta=$ $11.9^{\circ}$ corresponding to an interplanar distance of $0.78 \mathrm{~nm}$ and attributed to the oxide functional groups of the graphite basal plane during oxidation reaction, which are the responsibility of the consequent exfoliation as reported in other works, ${ }^{18}$ however oxidation is not totally achieved. In contrast, the chemically oxidized GO, only one single, sharp and intense diffraction peak appears at $10.6^{\circ}(d=0.82 \mathrm{~nm})$ confirming that chemical oxidation gives rise to a complete oxidation and exfoliation of graphite.

Both samples were also observed by TEM (Fig. 4) where a few exfoliated and typical transparent layers of graphene oxide (indicated as dashed regions) could be observed, but coexisting with other areas with stacked sheets (darkest regions) for both GO- $\left(\mathrm{NH}_{4}\right)_{2} \mathrm{CO}_{3}$ and the $\mathrm{GO}$ obtained by Marcano synthesis. This shows that GO obtained by electrochemistry and the chemical GO are similar.

\section{Synthesis of hollow ZnO microspheres and its functionalization with graphene oxide}

Representative SEM images are reported in Fig. 5. Additional images, used for the statistical analyses, are collected in the ESI. $\dagger$ The morphological analysis by SEM of the ZnO-carbon microspheres before calcination (Fig. 5a) shows a smooth surface with an average size for the carbon microspheres of 7.07 $\pm 2.5 \mu \mathrm{m}$. After calcination (Fig. $5 \mathrm{~b}$ ), the size of the microspheres decreases to $2.8 \pm 0.9 \mu \mathrm{m}$, the shell presents a thickness

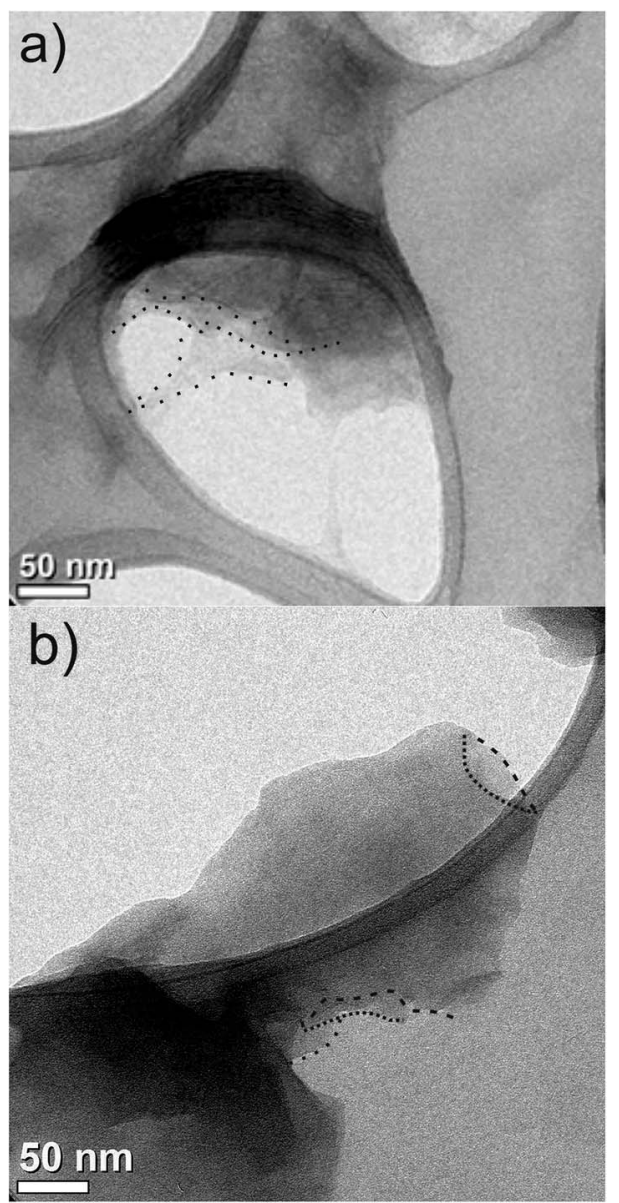

Fig. 4 TEM of (a) $\mathrm{GO}-\left(\mathrm{NH}_{4}\right)_{2} \mathrm{CO}_{3}$ and (b) GO respectively.

ranging from 30 to $200 \mathrm{~nm}$, where $\mathrm{ZnO}$ nanoparticles up to $70 \mathrm{~nm}$ can be observed. The opening of the microspheres is found between 500 and $900 \mathrm{~nm}$.

After functionalization with GO, the hollow microspheres surface is totally covered with graphene oxide, inclusive in the openings. It is worth mentioning that in the $\mathrm{GO}-\left(\mathrm{NH}_{4}\right)_{2} \mathrm{CO}_{3}-$

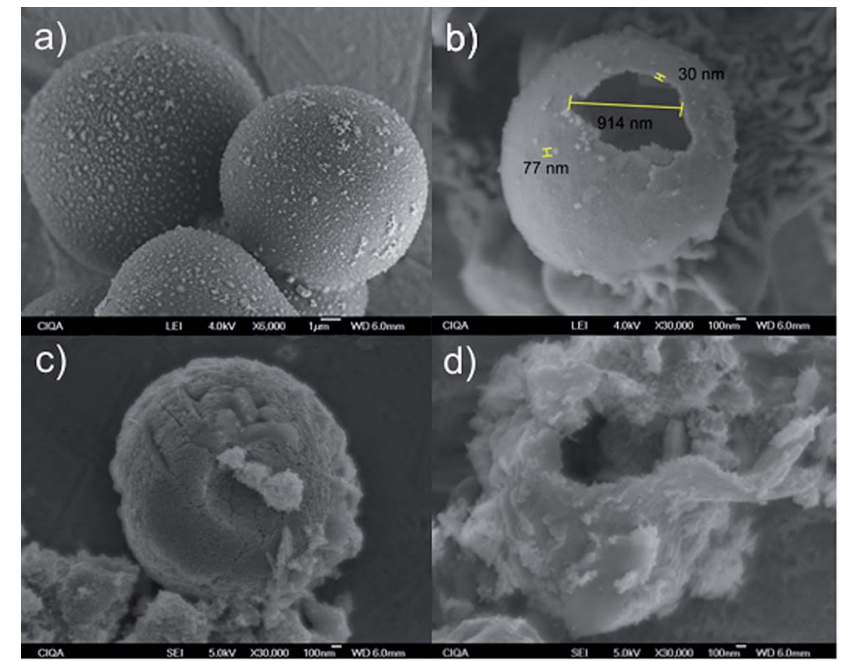

Fig. $5 \mathrm{ZnO}$-carbon microspheres before (a) and after (b) calcination. Hollow microspheres functionalized with $\mathrm{GO}$ (c) and $\mathrm{GO}-\left(\mathrm{NH}_{4}\right)_{2} \mathrm{CO}_{3}$, (d). 
$\mathrm{ZnO}$ case, the samples were slightly darker than those of GO$\mathrm{ZnO}$, this may be due to the presence of graphite.

The X-ray diffraction (Fig. 6) of carbon microspheres shows a broadband typical of an amorphous material with two main maximums at $2 \theta=17^{\circ}$ and $41^{\circ}$ corresponding to (002) and (101) lattice planes of carbon. ${ }^{27}$ After the calcination of the carbon microspheres, the diffractions at $2 \theta=31.8^{\circ}, 34.5^{\circ}, 36.1^{\circ}, 47.5^{\circ}$, $56.4^{\circ}, 62.9^{\circ}, 67.9^{\circ}$, and $69.0^{\circ}$ correspond to $\mathrm{ZnO}$ crystalline planes: (100), (002), (101), (102), (110), (103), (112) and (001) (JCPDS 36-1451, Fig. 6).

The materials functionalized with GO maintain the same diffraction peaks. Graphene diffractions can be observed only after magnification of the region $2 \theta=3-30^{\circ}$ (ESI $\dagger$ ).

In order to corroborate on the $\mathrm{GO}$ functionalization of $\mathrm{ZnO}$ hollow microspheres, XPS analysis was performed. Fig. 6 shows the survey spectrum for $\mathrm{ZnO}$, while those of $\mathrm{GO} / \mathrm{ZnO}$ and GO$\left(\mathrm{NH}_{4}\right)_{2} \mathrm{CO}_{3} / \mathrm{ZnO}$ are shown in the ESI. $\dagger$

The survey confirms the presence of adventitious carbon contamination ( $\mathrm{C}$ 1s), $\mathrm{O}$ and $\mathrm{Zn}$ signals correspond to $\mathrm{ZnO}$ hollow microspheres (Fig. 7). The same signals were observed after GO functionalization, evidently with many different relative intensities. Quantification of each peak gives rise to the elemental analysis reported in Table 1.

The results obtained after the deconvolution process performed on the high resolution XPS spectra of the $\mathrm{Zn} 2 \mathrm{p}_{3 / 2}, \mathrm{C} 1 \mathrm{~s}$ and $\mathrm{O}$ 1s signals, corresponding to the three samples, are shown in Fig. 8.

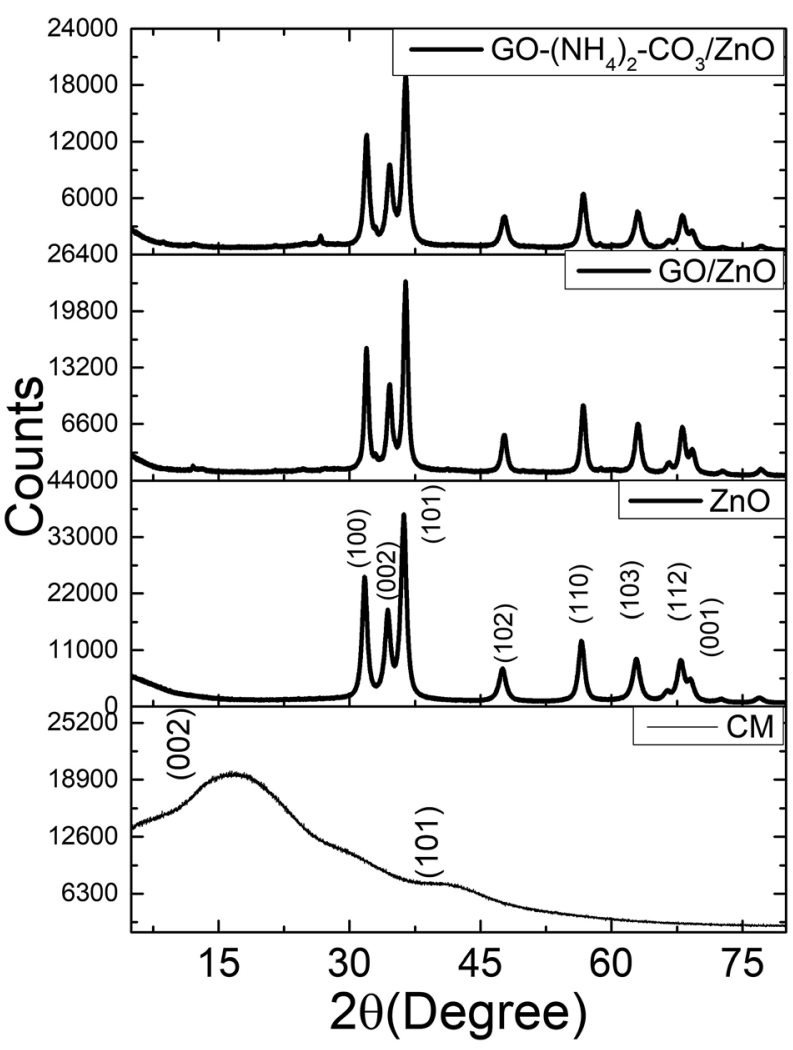

Fig. 6 Top: XRD of carbon microspheres (CM), ZnO hollow microspheres and $\mathrm{ZnO}$ hollow microspheres functionalized with chemical and electrochemical graphene oxide.

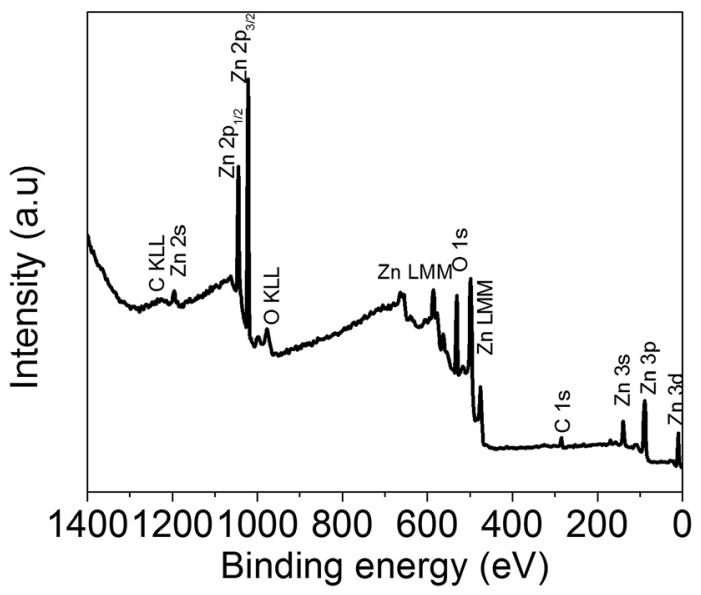

Fig. 7 XPS survey scan of ZnO hollow microspheres.

Table 1 Elemental composition as obtained by XPS survey spectra

\begin{tabular}{llll}
\hline Sample & C 1s (atm\%) & O 1s (atm\%) & Zn 2p (atm\%) \\
\hline $\mathrm{ZnO}$ & 14.2 & 50.3 & 35.4 \\
$\mathrm{GO} / \mathrm{ZnO}$ & 26.1 & 48.3 & 25.6 \\
$\mathrm{GO}-\left(\mathrm{NH}_{4}\right)_{2} \mathrm{CO}_{3} / \mathrm{ZnO}$ & 39.6 & 37.9 & 22.4
\end{tabular}

The shift of binding energy in the signal $\mathrm{Zn} 2 \mathrm{p}$ from 0.3 to $1.2 \mathrm{eV}$, in the samples demonstrates that the modification of ZnO hollow was successfully achieved by this route. The analysis of $\mathrm{C} 1 \mathrm{~s}$ confirms that the carbon present in the hollow ZnO sample was a residual carbon contamination, contrary to the $\mathrm{C} 1 \mathrm{~s}$, presented in the modified samples, for which the $\mathrm{Zn}-\mathrm{O}-\mathrm{C}$ bond could be identified, confirming the interaction and modification of the hollow spheres by the GO.

With respect to the $\mathrm{O} 1 \mathrm{~s}$ signal, the analysis indicates that the component $\mathrm{O}(\mathrm{I})$ can be attributed to the $\mathrm{Zn}-\mathrm{O}$ bonds, secondly the $\mathrm{O}(\mathrm{II})$ is associated with $\mathrm{O}^{2-}$ ions that are in oxygen-deficient regions within the $\mathrm{ZnO}$. As a result, changes in the intensity of this component may be in connection with the variations in the concentration of the oxygen vacancies, and the higher binding energy $\mathrm{O}$ (III), is usually attributed to chemisorbed or dissociated oxygen or $\mathrm{OH}$ species on the surface of the $\mathrm{ZnO} .^{28}$

The adsorption of GO on the hollow microspheres was also followed by UV-Vis spectroscopy, Fig. 9. The ZnO hollow microspheres exhibit a peak at $275 \mathrm{~nm}$ due to the plasmon resonance of $\mathrm{Zn}$ nanoparticles and a shoulder at around $370 \mathrm{~nm}$ attributed to the absorption of nanoparticles of $\mathrm{ZnO}$. These two absorptions persist in the functionalized microspheres as reported by other authors. ${ }^{29}$ Additionally, both GO/ $\mathrm{ZnO}$ and $\mathrm{GO}-\left(\mathrm{NH}_{4}\right)_{2} \mathrm{CO}_{3} / \mathrm{ZnO}$ present an absorption band at $240 \mathrm{~nm}$ attributed to the $\pi-\pi *$ transition of $\mathrm{C}=\mathrm{C}$ of graphene oxide. $^{30,31}$

This latter peak is, however, more pronounced for the chemical GO, in agreement with the higher oxidation as found previously by vibrational spectroscopy. 


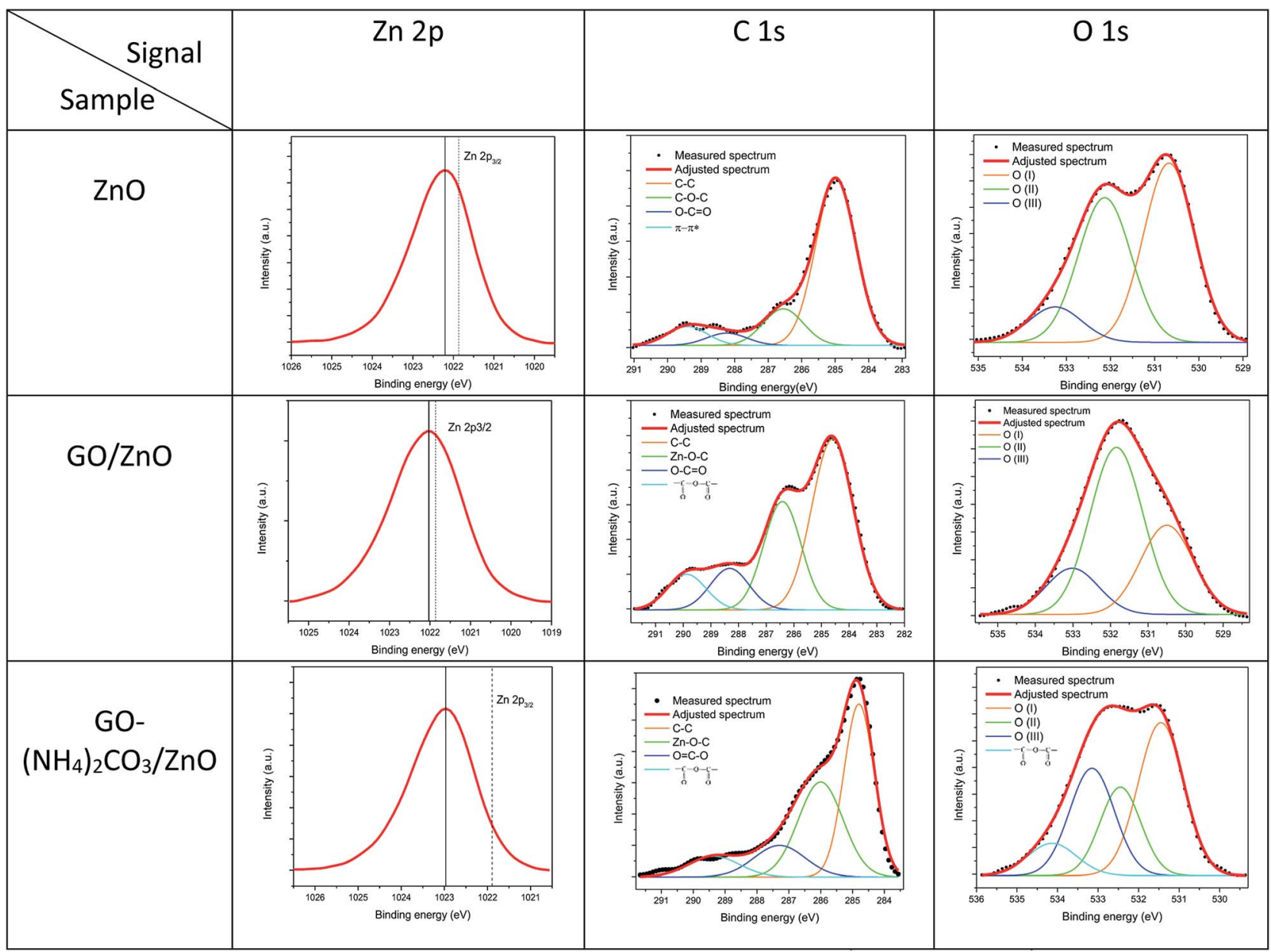

Fig. $8 \mathrm{HR} X P S$ analyses of the $\mathrm{Zn} 2 \mathrm{p}, \mathrm{C} 1 \mathrm{~s}$ and $\mathrm{O} 1 \mathrm{~s}$ signals, corresponding to the $\mathrm{ZnO}$ (hollow microspheres), $\mathrm{GO} / \mathrm{ZnO}$ and $\mathrm{GO}-\left(\mathrm{NH}_{4}\right)_{2} \mathrm{CO} 3 / \mathrm{ZnO}$ samples.

\section{Photocatalytic activity}

As previously mentioned, the photodegradation of salicylic acid was followed by UV-Vis spectroscopy. Fig. 10 reports the graphic of the concentration of salicylic acid $(C)$ normalized with respect

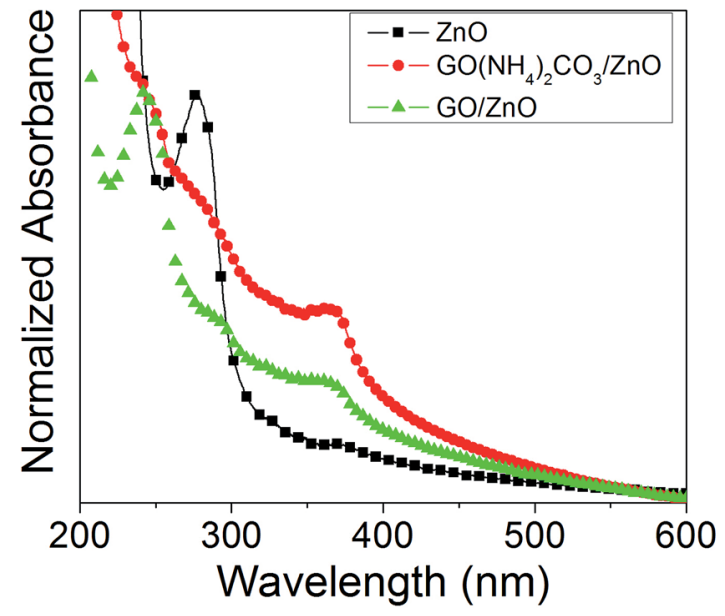

Fig. 9 UV-Vis absorption spectra of $\mathrm{ZnO}$ hollow microspheres (black square-line), $\mathrm{GO}-\left(\mathrm{NH}_{4}\right)_{2} \mathrm{CO}_{3}-\mathrm{ZnO}$ (red circle-line) and $\mathrm{GO}-\mathrm{ZnO}$ (green triangle-line) in water. to the initial value $\left(C_{0}\right) v s$. time, in the three different catalytic systems of this work: hollow $\mathrm{ZnO}$ microspheres without and with graphene oxide (electrochemically and chemically synthesized). It can be seen, that at $240 \mathrm{~min}$ of exposure, the concentration of salicylic acid decreases to $18 \%$ of its initial value for pristine $\mathrm{ZnO}$ microspheres, whereas the microspheres with graphene oxide give a decrease of up 29 and $27 \%$, for the chemical and electrochemical graphene oxide, respectively. The experimental data were fitted using the pseudo-first order kinetic equation: $\ln \left(C_{0} / C\right)=k t$ (Fig. 10, inserted). ${ }^{32}$ The salicylic acid degradation rate constants are $0.0015,0.0012$ and $0.0008 \mathrm{~min}^{-1}$ for $\mathrm{GO}-\mathrm{ZnO}, \mathrm{GO}-\left(\mathrm{NH}_{4}\right)_{2} \mathrm{CO}_{3}-\mathrm{ZnO}$ and $\mathrm{ZnO}$ respectively. The enhancement of the degradation constant observed for both graphene oxides can be explained on the basis of the photodegradation mechanism, Fig. 11. Under UV light but without catalyst or in presence of catalyst but without UV light, no reaction occurs, as the UV excitation is necessary for generating excitons in the semiconductor. Due to the electron donor character of salicylic acid and the electron acceptor nature of $\mathrm{ZnO}$, excitons are dissociated in the free charges: electrons $\left(\mathrm{e}^{-}\right)$and holes $\left(\mathrm{h}^{+}\right)$. As reported for other organic compounds in water, ${ }^{33}$ it is considered that in the valence band, holes generate $\mathrm{OH}$ radicals. 


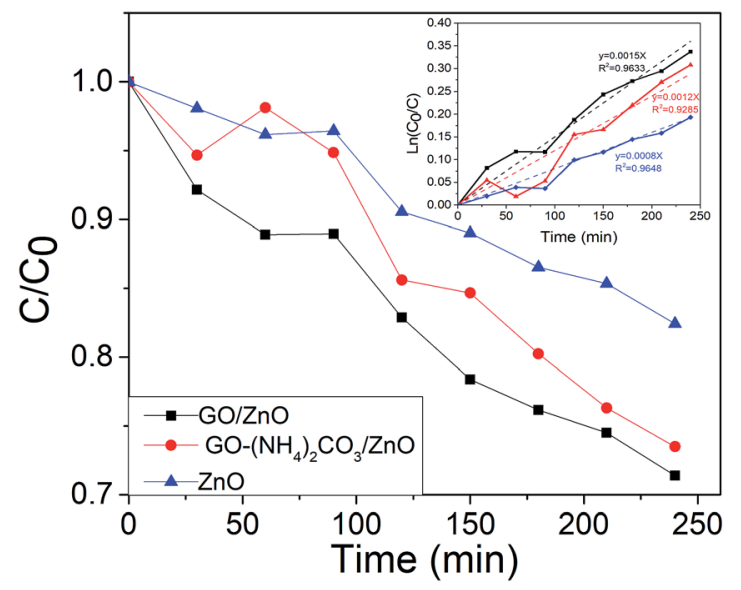

Fig. 10 Photocatalytic degradation of salicylic acid with hollow microspheres $\mathrm{ZnO}$ and hollow microspheres functionalized with $\mathrm{GO}$ and $\mathrm{GO}-\left(\mathrm{NH}_{4}\right)_{2} \mathrm{CO}_{3}$ under UV light irradiation. Insert the $\ln \left(\mathrm{C}_{0} / C\right)$ versus time curves of photodegradation of salicylic acid.

Conduction band electrons reduce dissolved oxygen forming hydrogen peroxide. Graphene oxide participates as an intermediary electron attractor to achieve the charges from $\mathrm{ZnO}$ through photoinduced electron transfer. Moreover, it is a semiconducting material, thus favouring the charge transport and reducing the possible $\mathrm{e}^{-} / \mathrm{h}^{+}$recombination. It is to be mention that a similar enhancement in the photocatalytic activity was found for graphene-ZnO hybrids. However, in that work $\mathrm{ZnO}$ particles were nanosized and graphene, not graphene oxide was used in order to obtain higher electrical conductivity with respect to pure $\mathrm{ZnO} \cdot{ }^{34}$ In this context, the almost equivalent behaviour of chemical and electrochemical graphene- $\mathrm{ZnO}$ samples indicates that the $\mathrm{GO}-\left(\mathrm{NH}_{4}\right)_{2} \mathrm{CO}_{3}$ exhibit a very good photocatalytic behaviour, despite the lower oxidation of graphite. Moreover, as it is obtained in a simpler, less toxic

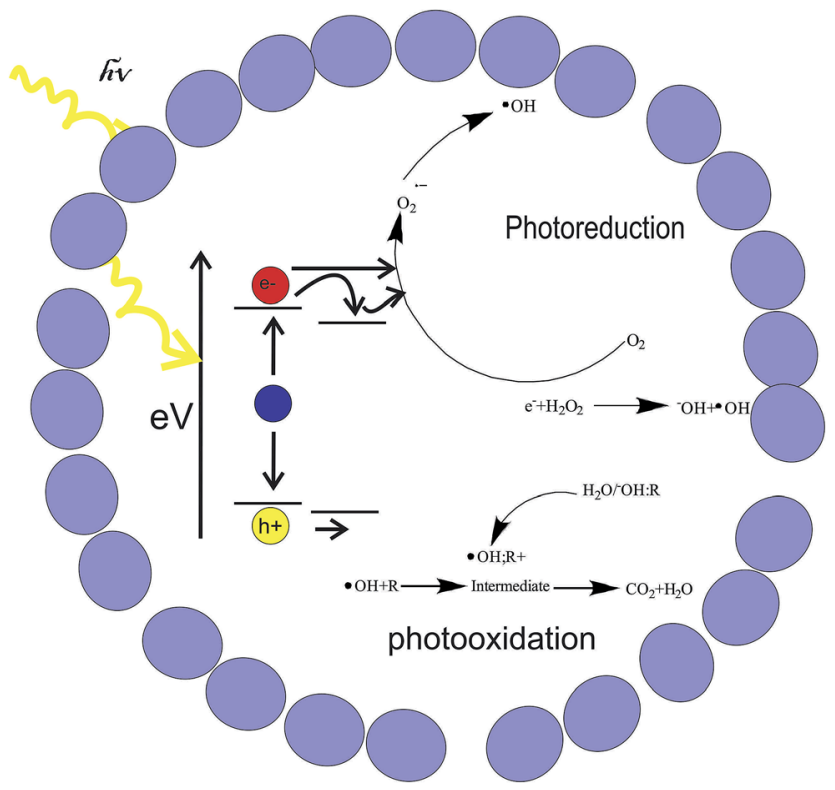

Fig. 11 Illustration of enhanced photocatalytic activity for GO-ZnO and $\mathrm{GO}\left(\mathrm{NH}_{4}\right)_{2} \mathrm{CO}_{3}-\mathrm{ZnO}$ Composites. method represents an eco-friendly alternative to classical chemical graphite oxide.

\section{Conclusion}

IR, Raman, and XRD spectroscopies reveal that electrochemical oxidation of graphite gives a partial oxidation with respect to classical chemical oxidation method. Nevertheless, its functionalization with hollow $\mathrm{ZnO}$ microspheres gives a composite material with similar photodegradation rate constants for salicylic acid as the corresponding with graphite oxide obtained with acids method, and even more effective compared with not functionalized $\mathrm{ZnO}$ microspheres. These results demonstrate that hollow ZnO microspheres functionalized with electrochemical graphite oxide represent an eco-friendly photocatalytic system for water treatment.

\section{Conflicts of interest}

There are no conflicts to declare.

\section{Acknowledgements}

The support of the CONACYT through Grant LNMG 293371 and LANIAUTO 294030 is greatly appreciated. Arxel de León thanks CONACyT-SENER (Mexico) for the postdoctoral fellowship.

\section{Notes and references}

1 N. K. Stamatis and I. K. Konstantinou, Occurrence and removal of emerging pharmaceutical, personal care compounds and caffeine tracer in municipal sewage treatment plant in Western Greece, J. Environ. Sci. Health, Part B, 2013, 48(9), 800-813.

2 C. Yu, K. Yang, Y. Xie, Q. Fan, C. Y. Jimmy, Q. Shu and C. Wang, Novel hollow Pt-ZnO nanocomposite microspheres with hierarchical structure and enhanced photocatalytic activity and stability, Nanoscale, 2013, 5(5), 2142-2151.

3 C. Yu, F. Cao, X. Li, G. Li, Y. Xie, C. Y. Jimmy, Q. Shu, Q. Fan and J. Chen, Hydrothermal synthesis and characterization of novel $\mathrm{PbWO}_{4}$ microspheres with hierarchical nanostructures and enhanced photocatalytic performance in dye degradation, Chem. Eng. J., 2013, 219, 86-95.

4 P. Cui, J. Wang, Z. Wang, J. Chen, X. Xing, L. Wang and R. Yu, Bismuth oxychloride hollow microspheres with high visible light photocatalytic activity, Nano Res., 2016, 9(3), 593-601.

$5 \mathrm{~J}$. Yu and G. Wang, Hydrothermal synthesis and photocatalytic activity of mesoporous titania hollow microspheres, J. Phys. Chem. Solids, 2008, 69(5-6), 11471151.

6 K. Qi, H. Qi, Y. Xie and Y. Wang, Enhanced Photocatalytic Performance of $\mathrm{ZnO}$ through Coupling with Carbon Materials, General Chemistry, 2017, 3(1), 113-117.

7 A. Jana, E. Scheer and S. Polarz, Synthesis of graphenetransition metal oxide hybrid nanoparticles and their 
application in various fields, Beilstein J. Nanotechnol., 2017, 8(1), 688-714.

8 W. Zhang, F. Liu, Q. Li, Q. Shou, J. Cheng, L. Zhang, B. J. Nelson and X. Zhang, Transition metal oxide and graphene nanocomposites for high-performance electrochemical capacitors, Phys. Chem. Chem. Phys., 2012, 14(47), 16331-16337.

$9 \mathrm{H}$. H. Mohamed, Sonochemical synthesis of $\mathrm{ZnO}$ hollow microstructure/reduced graphene oxide for enhanced sunlight photocatalytic degradation of organic pollutants, J. Photochem. Photobiol., A, 2018, 353, 401-408.

10 D. Zhang, Y. Zhao and L. Chen, Fabrication and characterization of amino-grafted graphene oxide modified ZnO with high photocatalytic activity, Appl. Surf. Sci., 2018, 458, 638-647.

11 D. C. Marcano, D. V. Kosynkin, J. M. Berlin, A. Sinitskii, Z. Sun, A. Slesarev, L. B. Alemany, W. Lu and J. M. Tour, Improved synthesis of graphene oxide, ACS Nano, 2010, 4(8), 4806-4814.

$12 \mathrm{~W}$. S. Hummers Jr and R. E. Offeman, Preparation of graphitic oxide, J. Am. Chem. Soc., 1958, 80(6), 1339.

13 B. C. Brodie II, On the atomic weight of graphite, Proc. R. Soc. London, 1860, 10, 11-12.

14 L. Staudenmaier, Verfahren zur darstellung der graphitsäure, Ber. Dtsch. Chem. Ges., 1898, 31(2), 1481-1487.

15 K. Kakaei and K. Hasanpour, Synthesis of graphene oxide nanosheets by electrochemical exfoliation of graphite in cetyltrimethylammonium bromide and its application for oxygen reduction, J. Mater. Chem. A, 2014, 2(37), 1542815436.

16 X. You, J.-H. Chang, B. K. Ju and J. J. Pak, An electrochemical route to graphene oxide, J. Nanosci. Nanotechnol., 2011, 11(7), 5965-5968.

17 E. Teran-Salgado, D. Bahena-Uribe, P. A. Márquez-Aguilar, J. L. Reyes-Rodriguez, R. Cruz-Silva and O. Solorza-Feria, Platinum nanoparticles supported on electrochemically oxidized and exfoliated graphite for the oxygen reduction reaction, Electrochim. Acta, 2019, 298, 172-185.

18 J. Qin, X. Zhang, C. Yang, M. Cao, M. Ma and R. Liu, ZnO microspheres-reduced graphene oxide nanocomposite for photocatalytic degradation of methylene blue dye, Appl. Surf. Sci., 2017, 392, 196-203.

19 S. M. Mousavi, A. R. Mahjoub and R. Abazari, Green synthesis of ZnO hollow sphere nanostructures by a facile route at room temperature with efficient photocatalytic dye degradation properties, RSC Adv., 2015, 5(130), 107378107388.

20 K. S. S. K. Muhamad, F. Mohamed, S. Radiman, A. Hamzah, S. Sarmani, K. K. Siong, M. S. Yasir, I. A. Rahman and N. R. A. M. Rosli, in Synthesis and characterization of exfoliated graphene oxide, AIP Conference Proceedings, AIP Publishing, 2016, p. 040013.

21 S. I. Javed and Z. Hussain, Covalently functionalized graphene oxide-characterization and its electrochemical performance, Int. J. Electrochem. Sci., 2015, 10, 9475-9487.
22 A. A. Dubale, W.-N. Su, A. G. Tamirat, C.-J. Pan, B. A. Aragaw, H.-M. Chen, C.-H. Chen and B.-J. Hwang, The synergetic effect of graphene on $\mathrm{Cu}_{2} \mathrm{O}$ nanowire arrays as a highly efficient hydrogen evolution photocathode in water splitting, J. Mater. Chem. A, 2014, 2(43), 18383-18397.

23 S. Stankovich, D. A. Dikin, R. D. Piner, K. A. Kohlhaas, A. Kleinhammes, Y. Jia, Y. Wu, S. T. Nguyen and R. S. Ruoff, Synthesis of graphene-based nanosheets via chemical reduction of exfoliated graphite oxide, Carbon, 2007, 45(7), 1558-1565.

24 J. P. Rourke, P. A. Pandey, J. J. Moore, M. Bates, I. A. Kinloch, R. J. Young and N. R. Wilson, The real graphene oxide revealed: stripping the oxidative debris from the graphene-like sheets, Angew. Chem., 2011, 123(14), 32313235.

25 M. Wojtoniszak, B. Zielinska, R. J. Kalenczuk and E. Mijowska, Photocatalytic performance of titania nanospheres deposited on graphene in coumarin oxidation reaction, Mater. Sci.-Pol., 2012, 30(1), 32-38.

26 M. Fathy, T. Abdel Moghny, M. A. Mousa, A.-H. A. A. ElBellihi and A. E. Awadallah, Absorption of calcium ions on oxidized graphene sheets and study its dynamic behavior by kinetic and isothermal models, Appl. Nanosci., 2016, 6(8), 1105-1117.

27 G. Krishnamurthy, R. Namitha and S. Agarwal, Synthesis of carbon nanotubes and carbon spheres and study of their hydrogen storage property by electrochemical method, Procedia Mater. Sci., 2014, 5, 1056-1065.

28 P.-T. Hsieh, Y.-C. Chen, K.-S. Kao and C.-M. Wang, Luminescence mechanism of $\mathrm{ZnO}$ thin film investigated by XPS measurement, Appl. Phys. A: Mater. Sci. Process., 2008, 90(2), 317-321.

29 M. H. Huang, S. Mao, H. Feick, H. Yan, Y. Wu, H. Kind, E. Weber, R. Russo and P. Yang, Room-temperature ultraviolet nanowire nanolasers, Science, 2001, 292(5523), 1897-1899.

30 G. Eda, Y. Y. Lin, C. Mattevi, H. Yamaguchi, H. A. Chen, I. S. Chen, C. W. Chen and M. Chhowalla, Blue photoluminescence from chemically derived graphene oxide, Adv. Mater., 2010, 22(4), 505-509.

31 Q. Lai, S. Zhu, X. Luo, M. Zou and S. Huang, Ultravioletvisible spectroscopy of graphene oxides, AIP Adv., 2012, 2(3), 032146.

32 W. Lu, S. Gao and J. Wang, One-pot synthesis of Ag/ZnO selfassembled 3D hollow microspheres with enhanced photocatalytic performance, J. Phys. Chem. C, 2008, 112(43), 16792-16800.

33 E. R. Carraway, A. J. Hoffman and M. R. Hoffmann, Photocatalytic oxidation of organic acids on quantum-sized semiconductor colloids, Environ. Sci. Technol., 1994, 28(5), 786-793.

$34 \mathrm{~T}$. Xu, L. Zhang, H. Cheng and Y. Zhu, Significantly enhanced photocatalytic performance of $\mathrm{ZnO}$ via graphene hybridization and the mechanism study, Appl. Catal., B, 2011, 101(3-4), 382-387. 